Available online on 15.09.2017 at http://jddtonline.info
Open Access to Pharmaceutical and Medical Research
(c)11-17, publisher and licensee JDDT, This is an Open Access article which permits unrestricted
noncommercial use, provided the original work is properly cited

Open $\odot$ Access

Review Article

\title{
A REVIEW ON THERAPEUTIC APPLICATIONS OF PHYTOSOMES
}

\author{
Chivte Prajkta* $^{* 1}$, Pardhi Vinal ${ }^{1}$, Joshi Vineeta ${ }^{1}$, Ajitha Rani $\mathbf{R}^{2}$ \\ ${ }^{1}$ National facility for Biopharmaceuticals, G N Khalsa College, Matunga, Mumbai-400019, India \\ ${ }^{2}$ Department of Chemistry, G N Khalsa College, Matunga, Mumbai-400019, India
}

\begin{abstract}
Ethnobotanical plants have been used in the treatment of various diseases since antiquity. The phytoconstituents have pharmacological activities such as anti-tumor, anti-inflammatory, antinociceptive, anti-obesity, thermoregulatory effects, cardioprotective, anti-asthmatic, anti-diabetic, anti-oxidant, hepatoprotective and potent CNS activities. Various drug delivery systems like liposomes, niosomes, transferosomes, ethosomes, phytosomes, colloidosomes etc. are developed in order to carry the drug to the site of action without its metabolism. The major concern with respect to phytoconstituents having different chemical nature is its bioavailability and effective dosage concentration which could be overcome by means of phytosome technology. Chemically, phytosomes are vesicles of phospholipids bonded with a hydrogen bond to phytochemical/s. The current review highlights the major therapeutic applications of phytosomes till date. The review discusses about a latest and novel drug delivery system viz. "Phytosome technology" whereby major emphasis has been provided on the different therapeutic applications of phytosomes and its crucial role in managing the conventional complications that are encountered for the delivery of phytoconstituents. Applications of phytosomes such as enhancing bioavailability, anti-cancer and anti-oxidant agent, transdermal delivery, wound healing capacity etc. have been discussed. The comparison between the therapeutic activities of free drug and its phytosomes indicates that the complex provides various advantages over the conventional treatments.
\end{abstract}

Keywords: Phytosome, Phytoconstituents, Bioavailability, Cancer, Anti-oxidant.

Article Info: Received 14 July, 2017; Review Completed 24 Aug, 2017; Accepted 24 Aug, 2017; Available online 15 Sep, 2017

\section{*Address for Correspondence}

Ms. Prajkta Chivte (MSc. By Research, Bioanalytical Sciences), National facility for Biopharmaceuticals, G N Khalsa College, Matunga, Mumbai-400019, India. Email: chivteprajkta@yahoo.in

Cite this article as:

Chivte P, Pardhi V, Joshi V, Ajitha RR, A review on therapeutic applications of phytosomes, Journal of Drug Delivery and Therapeutics. 2017; 7(5):17-21

DOI: http://dx.doi.org/10.22270/jddt.v7i5.1513

\section{INTRODUCTION:}

Over the last century, phyto-chemical science and phyto-pharmacological science established numerous plant compounds with various biological activities and health promoting benefits such as anti-mutagenicity, anti-carcinogenicity and anti-oxidative activity, for agerelated diseases namely memory loss, osteoporosis, diabetic wounds, immune and liver disorders, etc. Herbal medicines have been known since eons for their safety, efficacy, folk acceptability and fewer side effects. They are believed to have better compatibility with the human body since the chemical constituents present in them are a part of the physiological functions of living flora. $1,2,3$

However, most of these biologically active constituents of plants show poor bioavailability owing to their large molecular size, comprising of multiple rings, which cannot be absorbed by passive diffusion, or due to their poor lipid solubility; severely limiting the ability to pass across the lipid-rich biological membranes. ${ }^{4}$

Drug delivery systems (DDS) are capable of designing drugs with increased bioavailability and controlled delivery by minimizing drug degradation or presystemic metabolism of plant actives, also preventing the serious side effects resulting due to the accumulation 
of drugs to the non-targeted areas. It improves the ease of administration of such difficult phytochemicals to assimilate drugs in the pediatric and geriatric patients. 4

Control of the distribution of drug, in this novel drug delivery technology, is achieved by incorporating the drug (plant actives) in carrier system or in changing the structure of the drug at molecular level. This mechanism aids in increasing solubility, stability, protection from toxicity, pharmacological activity, improved tissue macrophage distribution and sustained delivery. ${ }^{6}$

Novel drug delivery system encompasses different types of pharmaceutical carriers such as polymeric micelles, particulate systems, macro- and micromolecules. Particulate type carrier, also known as colloidal carrier system, includes lipid particles, micro- and nanoparticles, micro- and nanospheres, polymeric micelles, and vesicular systems like liposomes, sphingosomes, niosomes, transfersomes, aquasomes, ufasomes, and so forth. The vesicular systems are highly ordered assemblies of one or several concentric lipid bilayer formed, when certain amphiphillic building blocks are confronted with water. These systems contribute in prolonging the existence of the drug in systemic circulation, reducing toxicity and delaying elimination of rapidly metabolizable drugs. ${ }^{7}$

Indena, an Italian pharmaceutical and nutraceutical company, first developed the complexation of plant extracts containing water-soluble constituents with phospholipids to improve their bioavailability. They patented the technology as 'PHYTOSOME®'. ${ }^{8}$ The term "phyto" means plant and "some" means cell like. Due to the creation of an $\mathrm{H}-$ bond between phospholipids and the phytoconstituents, phytosomes show better physical stability, enhancing absorption of hydrophilic polar phytoconstituents resulting in enhanced bioavailability and greater therapeutic benefits. ${ }^{9}$

Some phytoconstituents show more than one therapeutic activity, but also prove to be toxic at higher dosages. With the advent of phytosomes, it would rather alter its toxicity into effective aftermath even when used at high concentrations and thereby increasing its efficacy. ${ }^{10}$ Phytosomes, commonly known as herbosomes, is a novel phytolipid formulation technology attempting to break through the barriers put forth by the conventional drug delivery systems with respect to bioavailability and stability of plant-derived drugs.

With the advancements in science, the herbosomes have gained importance in various fields like pharmaceuticals, cosmeceuticals and nutraceuticals in preparing different formulations such as solutions, emulsion, creams, lotions, gels, etc. Products containing standardized polyphenolic extracts are now available in the market for clinical applications. Some of the popular ones include Casperome boswellia herbosome for treating inflammatory conditions, Ginkgo select herbosome as cognition enhancer and as anti-oxidant, Hawthorn herbosome for cardiovascular health, Sericoside herbosome as anti-wrinkle and tonic and 'Meriva' a curcumin herbosome for healthy joints. These herbosomes are significantly more bioavailable and hence therapeutically more effective than the standardized extracts or their conventional forms and are useful in various disorders. Several companies involved in production and marketing of herbosomal products are Indena, Jamieson natural resources, Thorne Research, Natural factors, and Natures herb ${ }^{1}$

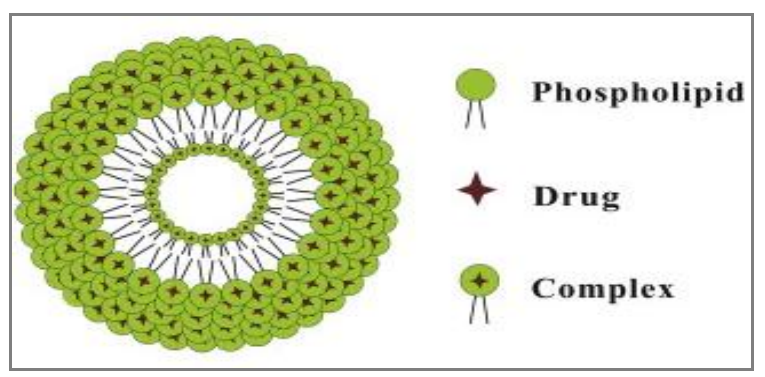

Figure 1: Diagrammatic representation of a phytosome

In this review, we have tried to present the subject of phytosome technology through a single dimension whereby major emphasis has been provided on the different therapeutic applications of phytosomes, encompassing in detail, the crucial role, this invention has played in managing the complications earlier encountered during plant derived drug delivery. This review is aimed to provide a general insight towards the effective implementation of phytosome as means of treating cancer, enhancing bioavailability, wound healing agent etc.

\section{APPLICATIONS:}

\section{Enhancing Bioavailability:}

Evodiamine, a quinoline alkaloid, (Evodia rutaecarpa) possess a multitude of pharmacological activities, such as anti-tumor, anti-inflammatory, anti-nociceptive, antiobesity, and thermoregulatory effects. Evodiamine has anti-tumor potential in a wide variety of tumor cells by inhibiting proliferation, inducing apoptosis and reducing invasion and metastasis. Phytosomes of Evodiamine proved to have higher in vitro dissolution rate, better absorption, longer action time and higher bioavailability. A prolonged action time and higher bioavailability was observed due to extended release of the drug from the phytosome. Moreover, these phytosomes might reduce the first-pass metabolism of Evodiamine by bypassing liver and therefore avoiding the direct contact of the drug with the hepatic metabolism enzymes. The bioavailability and $\mathrm{T}_{1 / 2}$ of Evodiamine was $1772.35 \mu \mathrm{g} \mathrm{h}^{-1} \mathrm{~L}^{-1}$ and 1.33 hours respectively. And the enhanced bioavailability and $\mathrm{T}_{1 / 2}$ with phytosome was $3787.24 \mu \mathrm{g} \mathrm{h}^{-1} \mathrm{~L}^{-1}$ and 2.07 hours respectively. ${ }^{11}$

A European patent by 'Indena S.P.A.' relates to novel phospholipids complexes of olive fruits or leaf extracts having improved bioavailability. The Oleaselect phytosomes were synthesized using purified olive fruit extract possessing anti-hypertensive, diuretic, antiatherosclerotic, anti-oxidant and hypoglycemic properties, on account of its extensive phytochemical profile. The group treated with Oleaselect phytosomes showed a higher percentage of HT (hydroxytyrosol) and HVAlc (homovanillyl alcohol) indicating increase in oral bioavailability as compared to the group treated 
with uncomplexed extract. A dose-related reduction of isoprostanes excretion was also observed. ${ }^{12}$

Ellagitannins belongs to a class of polyphenols which possess antioxidant mechanisms against cardiovascular disorders, cancers, and also wound healing, due to their antibacterial and anti-viral activities. The $\mathrm{P}_{\text {app }}$ (Apparent permeability) values for ellagitannins and its phytosomes by Everted gut sac technique were found to be 60 and $135 \mathrm{~nm} / \mathrm{s}$ respectively. This showed that the apparent permeability of ellagitannins increased almost two times on complexation with Phosphatidylcholine. ${ }^{13}$

The factors responsible for poor oral bioavailability of the phytoconstituents could be the dosage form, low concentrations available after chemical degradation, physical inactivation and elimination through the gut wall and liver. ${ }^{14}$ However, the enhanced bioavailability of phytosomes administered through oral route is a result of increased hydrophilicity, solubility, decreased hepatic metabolism and enriched drug absorption in the systemic circulation.

\section{Antioxidant properties}

S. Moscarella et al 1993 studied the antioxidant and free radical scavenging activity of Silipide which is phytosome of Silybum marianum plant against liver oxidative damage induced by $\mathrm{CCl}_{4}$ and paracetamol (high dosages) in rats. The mechanism by which Silipide protects hepatocytes against oxidative damage may be through inhibition of lipid peroxidation by scavenging reactive oxygen species. S. Moscarella et al 1993 continued the studies on patients with Hepatitis B Virus and Hepatitis C Virus with Silipide for 2 months and determined that it remarkably reduced liver function parameters related to hepatocyte necrosis or increased cell membrane permeability. Serum Malondialdehyde levels of patients treated with silipide showed a remarkable reduction (36\%) after 2 months of therapy suggesting that it possesses anti-lipoperoxidant activity against free radical attack in humans. ${ }^{15}$

A physically stable phytosomal formulation of Quercetin was prepared with higher encapsulation efficiency and physical stability to improve its efficacy in intestinal absorption and its preservation from oxidation in foodstuffs. ${ }^{16}$

One of the latest studies published in 2014 by B. Demir et al. 2014, talks about metal phytosome synthesized by encapsulation of extract of Calendula officinalis and its anti-oxidative effect. Reactive oxygen species analysis was performed using in vitro cell-based anti-oxidant assay on Vero cell lines. The outcome of this study depicted that the cell viability was approximately $35 \%$ and $81 \%$ of the plant extract and Au-loaded phytosome respectively. ${ }^{17}$

\section{Hepato-Protective:}

The leaf extracts of Ginkgo biloba (Family: Ginkgoaceae) have been found to possess cardioprotective, anti-asthmatic, anti-diabetic, antioxidant, hepatoprotective and potent CNS activities. The study demonstrated that phytosomes of G. biloba (200 $\mathrm{mg} / \mathrm{kg}$ ) significantly alleviated isoproterenol-induced myocardial necrosis. Histopathological examination of the myocardium further confirmed the cardioprotective effects of phytosomes. Decreased myocardial necrosis (as evidenced by reduced AST, LDH and CPK release and histoarchitectural changes) and augmentation of endogenous antioxidants, all contribute to its cardioprotective effect. ${ }^{18}$ Literature also reveals that clinical hepatitis occurs in $1.1 \%$ of adults receiving Rifampicin treatment. S.R. Naik et al. 2008 investigated about the protective effects of Ginkgoselect Phytosome ${ }^{\circledR}$ on Rifampicin (RMP) induced hepatotoxicity and the probable mechanism(s) involved in this protection in rats. The present findings suggest that the hepatoprotective effect of Ginkgo select Phytosome ${ }^{\circledR}$ in RMP induced oxidative damage may be linked to its antioxidant and free radical scavenging activity. ${ }^{19}$

Mangiferin (MF) showed potent scavenging activity on Diphenyl-1-picrylhydrazyl (DPPH) radicals which stimulates liver regeneration in various liver injuries. $E x$ vivo study showed significant increased absorption of MF from MF herbosomes as compared to plain MF. The hepatoprotective potential of MF herbosomes evaluated by in vivo study revealed significantly decreased levels of serum glutamate oxaloacetate transminase (SGOT), serum glutamate pyruvate transaminase (SGPT), total bilirubin, and alkaline phosphatase (ALP) in MF herbosomes as compared to plain MF. MF herbosomes also showed significantly decreased level of malonyl dehydrogenase along with increased levels of reduced glutathione, superoxide dismutase (SOD) and catalase as compared to plain MF which was also comparable to the standard drug, Silymarin (SL). ${ }^{20}$

The traditional uses of Andrographolide (AN) obtained from Andrographis paniculata Linn include treatment of fever, inflammation, common cold, tonsilitis, pharyngitis, laryngitis, pneumonia, tuberculosis, pyelonephritis and hepatic impairment etc. The equimolar dose of drug shows lesser absorption and elevated levels of SGOT and SGPT in serum as compared to its phytosome dose indicating its hepatoprotective nature. ${ }^{21}$

\section{Cancer treatment:}

The chemical components like flavones, isoflavones, flavonoids, anthocyanins, coumarins, lignins, catechins, and isocatechins of medicinal plants mainly possess antioxidant properties that contribute to their anticancer potential. However, a few plant-based compounds are toxic at higher concentrations and induce certain side effects. The already available expensive conventional therapies for cancer like chemotherapy and radiotherapy have a number of side effects such as myelosuppression and neurological, cardiac, pulmonary, and renal toxicity, which pose serious harm to the quality of life. ${ }^{22}$ The entrapment of these plant derived drugs with the aid of bipolar moiety to enhance its solubility, dispersibility and permeability and thus prove to be a potent anticancer agent.

S. Shalini et al. 2015 researched on methanolic extract of Terminalia arjuna bark and its phytosome to investigate its antiproliferative activity on human breast 
cancer cell line MCF-7 by MTT assay by comparing its activities with Quercetin and its phytosomes. The $\mathrm{IC}_{50}$ values of the extract and its phytosome were $25 \mu \mathrm{g} / \mathrm{ml}$ and $15 \mu \mathrm{g} / \mathrm{ml}$ respectively which suggests that they exert more antiproliferative effect as compared to free drug. ${ }^{23}$

D. Gallo et al. 2003 aimed to assess growth inhibitory effect of Silipide (Silybin complex) against human ovarian cancer (HOC) in vivo. The downregulation and upregulation of Vascular Endothelial Growth Factor (VEGF) and Angiopoietin-2 respectively indicates the antiangiogenic activity. HPLC analysis of free Silybin levels was found to be $7.0 \mathrm{mg} / \mathrm{ml}$ and $183.5 \mathrm{ng} / \mathrm{g}$ tissue in the plasma and tumor samples, respectively. No significant differences were seen in human VEGF levels in Silybin treated cells and vehicle blank. But a consistent decrease in VEGF concentration was observed in the tumor specimens treated with Silipide. The major finding of this study was to affirm Silipide as good candidate for the management of recurrent ovarian cancer. ${ }^{24}$

Narges Mahmoodi et al. 2014 worked with Silybin and its phytosomes to study the expression levels of estrogen receptor $\alpha(\mathrm{ER} \alpha)$ overexpressed in breast cancer, which is responsible for tumor growth enhancement, and is a prognostic and predictive factor. Silybin (silibinin) (Silybum marianum) is a natural polyphenol with high antioxidant and anti-cancer properties which blocks VEGF, EGFR, COX-2 and TNF. The comparison of silybin and silybin-phosphatidylcholine by MTT assay indicates that all silybin-phosphatidylcholine doses had 2.5-3 times more inhibitory effect on cell growth than the same silybin doses in the T47D cell line. The results for 48 hours indicated all doses significantly down regulated ESR. (Erythrocyte sedimentation rate) ${ }^{25}$

M. Sabzichi et al. 2014 explored the previously studied role of Nrf2 (Nuclear factor erythroid 2-related factor 2) in resistance to chemotherapeutic agents in different types of cancer. In this study, M. Sabzichi et al. 2014, used Luteolin phytosomes to sensitize cancer cells MDA-MB 231cells (human breast cancer cell line) to chemotherapeutic agent Doxorubicin by downregulating the Nrf2 expression. The probable mechanism of action of Luteolin would be such that due to presence of Luteolin phytosomes the action of detoxifying enzymes and transporters could not act on Doxorubcin, as a result cells becomes sensitive to the drug. ${ }^{26}$

\section{Transdermal application}

Malay K Das et al. 2104, investigated about Rutin, one of the most common flavonoid (Ruta graveolens) used to treat capillary fragility, hypertension, ultraviolet radiationinduced cutaneous oxidative stress, hepatic and blood cholesterol, cataract, cardiovascular disease and possesses antioxidant, anti-inflammatory, antithrombotic, antineoplastic, and antiplatelet activity. It was observed that the Rutin phytosomes were better able to penetrate the impermeable stratum corneum than its free form. Skin uptake of Rutin phytosomes was $33 \pm 1.33 \%$ whereas that of Rutin was $13 \pm 0.87 \%$. ${ }^{27}$

The phytosomal complex of saponins and plant extracts (Panax ginseng M.) proved to be more active in vasal protection, capillary permeability, protection against UV radiation. Also helpful in preparation of dermatological and cosmetic pharmaceutical formulations, exhibiting moisturizing effect on the cutis making it more elastic due to a fibroblastic stimulation at the dermal level, with an increase in proteoglycan and collagen synthesis. The above mentioned compositions can in particular be used for oral administration in the form of tablets, capsules, syrups, granules, solutions (containing $1-500 \mathrm{mg}$ dose of the complex) for treating conditions of inflammation, altered capillary fragility and permeability and, in general, in all the fields in which an activity of the saponins is recognized at present. ${ }^{28}$

\section{Wound healing}

A. Mazumder et al. 2016 studied the wound healing activity of Sinigrin, one of the major glucosinolates occurring in the plant of Brassicaceae family, when evaluated as alone and as a phytosome complex on HaCaT cells. The sinigrinphytosome complex shows complete recovery of wound $(100 \%)$ whereas the phytoconstituent alone displayed only $71 \%$ healing. Moreover, sinigrin phytosomes also show enhanced anti-cancer activity on the A- 375 melanoma cells. 10

S. Lakshmi Devi et al. 2012, researched on the comparative effects of ethanolic extracts of Wrightia arborea leaves and its phytosomes. The phytosomes exhibited about $90.40 \%$ healing while the ethanolic extract alone could heal only $65.63 \%$ of the wound. ${ }^{29}$ As discussed earlier, the study of Demir et al. 2014, the formulated vesicles also exhibited enhanced wound healing activity $(58.7 \%)$ and penetration in the cells, accumulating around nucleus. ${ }^{17}$

Limitations of phytosome:

Phytosomes, despite of having numerous advantages as drug delivery system, are not prevalent in the market. Yamila B. Gándola et al. 2014, mentioned that phospholipids (lecithin) can induce proliferation on MCF-7 breast cancer cell line. ${ }^{30} \mathrm{~A}$ major drawback of phytosome could be leaching of the phytoconstituents off the 'some' which reduces the desired drug concentration indicating their unstable nature.

\section{CONCLUSION:}

An attempt was made to explore the ongoing research with respect to phytosomes and its applications such as wound healing, anti-oxidant, anti-cancer activities, etc. Phytosome technology proves to be efficient drug delivery system and has higher significance than the free drug. The information gathered herein will be useful for the researchers who wish to explore a vesicular drug delivery system which encompasses effective drug on target site without its metabolism. Phytosome technology should be explored for the treatment of other neurodegenerative, cardio-vascular and auto-immune diseases and skin-related disorders. Although, various phytosome products are available in the market, yet there are many other phytoconstituents which have remarkable ability to treat life threatening diseases, have not been implemented into phytosome technology. Further research can be done to develop highly targetspecific phytosomes.

\section{Conflict of interest statement}

We declare that we have no conflict of interest. 


\section{REFERENCES:}

1. More Minakshi S, Shende Mulchand A, Kolhe Deul B, J. N. M., Herbosomes: herbo-phospholipid complex an approach for absorption enhancement, Int. J. Biol. Pharm. Res., 2013, 4(9), 634-639.

2. Kuamwat Radhey Shyam, Mruthunjaya K, G. M. K.,Preparation, characterisation and antioxidant activities of gallic-acid phospholipid complex, Int. J. Res. Pharm. Sci.,2012, 2, 138-148.

3. Pathak K. \& Das R. J., Herbal Medicine-A Rational Approach in Health Care System, Int. J. Herb. Med. IJHM,2013, 1(13), 86-89.

4. Tripathy S, Patel DK, Barob L, Naira SK, A review on phytosomes, their characterization, advancement \& potential for transdermal application, Journal of Drug Delivery and Therapeutics, 2013; 3(3):147-152

5. Jing Li, Xulin Wang, Ting Zhang, Chunling Wang, Zhenjun Huang, Xiang Luo, Yihui Deng, A review on phospholipids and their main applications in drug delivery systems, Asian J. Pharm. Sci.,2104, 10(2), 81-98.

6. Anju Dhiman, Arun Nanda, S. A., Novel Herbal Drug Delivery System (NHDDS): the need of hour, Int. Conf. Environ. Energy Biotechnol,2013, 51(26), 139-142.

7. Jadhav S. M., Morey P., Karpe M. M. \& Kadam V., Novel vesicular system: An overview, J. Appl. Pharm. Sci.,2012, 2(1), 193-202.

8. Saraf S., Khan, J., Alexander A., Ajazuddin \& Saraf S., Recent advances and future prospects of phyto-phospholipid complexation technique for improving pharmacokinetic profile of plant actives, J. Control. Release, 2013,168(1), 50-60.

9. Amit P., Tanwar Y. S., Rakesh S. \& Poojan P., Phytosome Phytolipid Drug Delivery System for Improving Bioavailability of Herbal Drug, J. Pharm. Sci. Biosci. Res., 2013, 3(2), 51-57.

10. Mazumder A., Dwivedi A., Du Preez J. L. \& Du Plessis J., In vitro wound healing and cytotoxic effects of sinigrinphytosome complex, Int. J. Pharm., 2016, 498, 283-293.

11. Qunyou Tan, Shan Liu, Xueliang Chen, Mingjun Wu, Hong Wang, Huafeng Yin, Dan He, Huarong Xiong, and Jingqing Zhang, Design and evaluation of a novel evodiaminephospholipid complex for improved oral bioavailability, AAPS PharmSciTech, 2012, 13(2), 534-547.

12. Franceschi, Federico, Giori, A., Phospholipid compleses of olive fruits or leaves extracts having improved bioavailability, 2006, 1, 1-8.

13. Vora A. K., Londhe V. Y. \& Pandita N. S., Preparation and characterization of standardized pomegranate extractphospholipid complex as an effective drug delivery tool, J. Adv. Pharm. Technol. Res.,2015, 6(2), 75-80.

14. Kwan K. C., Oral bioavailability and first-pass effects, Drug Metab. Dispos.,1997, 25(12), 1329-1336

15. Moscarella S. et al., Therapeutic and antilipoperoxidant effects of silybin-phosphatidylcholine complex in chronic liver disease: Preliminary results, Curr. Ther. Res., 1993, 53(1), 98102.

16. Rasaie S., Ghanbarzadeh S., Mohammadi M. \& Hamishehkar H., Nano Phytosomes of Quercetin: A Promising Formulation for Fortification of Food Products with Antioxidants. Pharm. Sci., 2014, 20, 96-101.

17. B. Demir, F. B. Barlas, E. Guler, P. Z. Gumus, M. Can, M. Yavuz, H. Coskunolbef and S. Timur, Gold nanoparticle loaded phytosomal systems: synthesis, characterization and in vitro investigations, RSC Adv., 2014,4, 34687.

18. Panda V. S. \& Naik S. R., Cardioprotective activity of Ginkgo biloba Phytosomes in isoproterenol-induced myocardial necrosis in rats: A biochemical and histoarchitectural evaluation, Exp. Toxicol. Pathol., 2008, 60, 397-404

19. Naik S. R. \& Panda V. S., Hepatoprotective effect of Ginkgoselect Phytosome ${ }^{\circledR}$ in rifampicin induced liver injurym in rats : Evidence of antioxidant activity, Fitoterapia, 2008, 79, $439-445$

20. Jain P. K., Kharya M. \& Gajbhiye A., Pharmacological evaluation of mangiferin herbosomes for antioxidant and hepatoprotection potential against ethanol induced hepatic damage, Drug Dev. Ind. Pharm., 2013, 39(11), 1840-1850.

21. Jain P. K., Khurana N., Pounikar Y., Gajbhiye A. \& Kharya M. D., Enhancement of absorption and hepatoprotective potential through soya-phosphatidylcholine-andrographolide vesicular system, J. Liposome Res., 2013, 23(2), 110-8.

22. Raina H., Soni G., Jauhari N., Sharma N. \& Bharadvaja N., Phytochemical importance of medicinal plants as potential sources of anticancer agents, Turk. J. Botany, 2014, 38, 10271035.

23. Shalini S., Kumar R. R. \& Birendra S., Antiproliferative effect of Phytosome complex of Methanolic extact of Terminalia arjuna bark on Human Breast Cancer Cell Lines (MCF-7), Int J Drug Dev Res.,2015, 7(1), 173-182.

24. D. Galloa, S. Giacomellia, C. Ferlinia, G. Raspaglioa, P. Apollonioa, S. Prisleia, A. Rivab, P. Morazzonib, E. Bombardellib, G. Scambia, Antitumour activity of the silybinphosphatidylcholine complex , IdB 1016 , against human ovarian cancer, Eur. J. Cancer, 2003, 39, 2403-2410.

25. Narges Mahmoodi, Nasrin Motamed, Seyed Hassan Paylakhi, The Comparison of The Effects of Silybin and SilybinPhosphatidylcholine on Viability and ESR Expression in Human Breast Cancer T47d cell line, Cell J., 2014; 16(3):299308.

26. Mehdi Sabzichi, Hamed Hamishehkar, Fatemeh Ramezani, Simin Sharifi, Maryam Tabasinezhad, Mohammadbagher Pirouzpanah1, Parisa Ghanbari3, Nasser Samadi., Luteolinloaded phytosomes sensitize human breast carcinoma MDAMB 231 cells to doxorubicin by suppressing Nrf2 mediated signalling, Asian Pacific J. Cancer Prev., 2014; 15(13):53115316

27. Das M. K. \& Kalita B., Design and Evaluation of PhytoPhospholipid Complexes (Phytosomes) of Rutin for Transdermal Application, J. Appl. Pharm. Sci., 2014; 4(10):5157.

28. Bombardelli, Patri, P. Complexes of saponins with phosphlipids and pharmaceutical and cosmetic compositions containing them. 9 (1988).

29. Lakshmi Devi, S. \& Divakar M. C. Wound healing activity studies of Wrightia arborea phytosome in rats. Hygeia J. drugs Med. 2012; 4(2):87-94

30. Yamila B. Gándola, Sebastián E. Pérez, Pablo E. Irene,Ana I. Sotelo, Johanna G. Miquet, Gerardo R. Corradi, AdrianaM. Carlucci, and LorenaGonzalez1. Mitogenic effects of phosphatidylcholine nanoparticles on MCF-7 breast cancer cells. Biomed Res. Int. 2014. 